\title{
SEGURIDAD JURÍDICA Y SISTEMA CAUTELAR
}

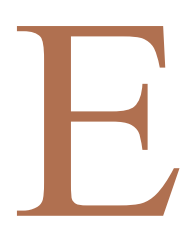

n una monografía reciente Peter Häberle ha asignado al estilo literario jurídico de la recensión el papel de factor y espejo de la evolución del derecho (Faktor und Spiegel der Rechtsentwicklung). Para cumplir esa tarea, esta manifestación de la cultura científico jurídica, debe contribuir a una triple función: informativa de las novedades bibliográficas relevantes; receptora de las orientaciones y cambios de rumbo predominantes en la teoría y/o práctica del derecho; y productora, a su vez, de tesis y concepciones a través de la prolongación o la crítica de las ideas recensionadas. ${ }^{1}$ Sabedor de la dificultad que entraña el logro de esa tercera función, no quisiera que ello me desalentara del empeño por cumplir las otras dos respecto a una obra que estimo digna de ser conocida y analizada.

El estudio que da pauta a este comentario $^{2}$ es fruto de una labor demorada en pacientes años de investigación, que tuvo su primer reconocimiento como tesis doctoral defendida con la máxima calificación en la Facultad de Derecho de la Universidad de Barcelona en 1988. Galardonada luego con el Premio Falguera, distinción quinquenal instituida por el Colegio Notarial de Barcelona, al que pertenece José Luis Mezquita del Cacho. Establecidas, de forma urgente, las dos coordenadas identificadoras básicas que exige cualquier comentario: la obra y su autor, procede iniciar su exploración.

$$
* * *
$$

${ }^{1}$ P. Häberle, «Wissenschaflitche Zeitschriften als Aufgabenfeld juristischen Rezensionswesens», en el vol. Verfassungsrecht und Völkerrecht. Gedächtnisschrift für Wilhelm Karl Geck, ed. a cargo de W. Fiedler y G. Ress, Carl Heymanns Verlag, Köln-Berlin-Bonn-München, 1989, págs. 277 y sigs.

2 J. 1. Mezquita del Cacho, Seguridad jurídica y sistema cautelar. 1. Teoría de la seguridad jurídica; 2. Sistema español de Derecho cautelar, Barcelona, Editorial Bosch, 1989, vol. 1, 396 págs.; vol. 2, 547 págs. 
Los dos volúmenes que, por el momento, ${ }^{3}$ conforman la obra dividen su contenido en función del análisis del Sistema o Teoría General de la seguridad jurídica, objeto del tomo primero; y el desenvolvimiento del sistema español de protección preventiva de la seguridad jurídica privada, o sea, de nuestro Sistema de Derecho cautelar, problemática que constituye el núcleo informador del tomo segundo.

El primer volumen se articula a su vez, en torno a dos grandes cuestiones: 1. a) $\mathrm{La}$ consideración de la seguridad jurídica como valor y como principio irrenunciable de los ordenamientos jurídicos democráticos. En función de ese planteamiento se estudian sucesivamente la formación histórica y delimitación conceptual de la idea de seguridad jurídica (págs. 15-109); el papel de tal noción en el ámbito de la división de los poderes públicos y de la interdicción de su arbitrariedad (págs. 109-169); su incidencia en la legalidad y en la tensión entre ésta y la libertad (págs. 169-198); así como sus relaciones con el valor de la justicia y con la Dogmática jurídica (págs. 199-236). 2. ${ }^{\text {) }}$ ) El estudio de las proyecciones de esa función de la seguridad en la esfera del Derecho privado, precisando los rasgos principales que deben concurrir en ella para el logro de su finalidad: dinamismo frente a estatismo, y dimensión preventiva, en cuanto forma o procedimiento institucional de garantía (págs. 237-398).

El volumen segundo se ocupa del desenvolvimiento del marco teórico del Sistema general de seguridad jurídica, diseñado en el primero, en el ordenamiento cautelar español. A partir de la concepción del Sistema cautelar como el conjunto de recursos de acción para la normalidad cotidiana de la aplicación del Derecho en la esfera privada, que se materializan en la investidura formal como institución suplente y presuntiva de la titularidad, se establecen los rasgos de su formación, evolución en el Derecho comparado (págs. 1-32). Un apartado importante de la investigación se dirige a establecer el status científico y la naturaleza jurídica del Sistema cautelar (págs. 33-210). Son objeto de una exposición pormenorizada las distintas funciones en que se desglosa la seguridad preventiva (págs. 211-287), así como las instituciones normativo-conceptuales y operativas a través de las que dichas funciones se realizan (págs. 287-511). La obra se cierra

${ }^{3}$ El Autor revela en el pórtico de su obra su propósito de proseguirla con sucesivos tomos dedicados al Amparo Cautelar a cargo de jueces y fiscales, asistidos de secretarios juridiciales; a la función cautelar documentadora a cargo del notariado; y, por último, a la publicidad cautelar de los registros. 
con un Apéndice, balance de cuanto ha representado históricamente el Sistema cautelar español y programa de sus posibles aportaciones al ordenamiento eurocomunitario (págs. 515-547). La larga experiencia de nuestro sistema cautelar, síntesis armonizadora de múltiples instituciones provenientes de diferentes familias jurídicas; así como la convivencia secular de instituciones comunes y forales en nuestro marco jurídico-político, hacen del sistema español un modelo que, en opinión del Autor, no debiera ser desatendido en la conformación del futuro sistema de seguridad de las relaciones jurídicas privadas de la Europa unida.

Constituye el nervio informador de la faceta constructiva de la investigación objeto de este comentario la observación, recurrente a lo largo de sus páginas, de que se debe al penetrante sentido jurídico de los romanos la consciencia de que no es posible concebir las fuentes del Derecho al margen de los cauces que las alimentan. Para designar a esas minas o filones utilizaron las expresiones, referidas a otros tantos órdenes de acción jurídica: respondere, postulare $y$ cavere. ${ }^{4}$ Con el primer término se aludía a la actividad dictaminadora, embrión de lo que en el decurso del tiempo sería el quehacer científico de la Dogmática. En el segundo se incluían las técnicas de argumentación forense de las que emergerán la jurisprudencia y las garantías procesales. El cavere significaba la acción precautoria, preventiva o cautelar de actos y contratos jurídicos, para evitar o reducir controversias y, en última instancia, para facilitar su resolución.

Nos hace observar el Autor que, en la trayectoria histórica, Estado y Sociedad han mantenido un acuerdo básico sobre el respondere y el postulare. Ambas instituciones han tendido a que el Derecho respondiera a criterios de racionalidad abstracta, pero adecuándose a las exigencias concretas del caso y dentro de un procedimiento que garantizara los intereses de las partes. En cambio, el devenir registra una secular contienda Estado/Sociedad en lo referente al cavere. La filosofía estatal al respecto cifraba en el intervencionismo público en la formalización de actos y contratos la garantía de seguridad y objetividad. La de la

${ }^{4}$ Respondere, postulare, cavere, fue precisamente el título elegido por José Luis Mezquita del Cacho para sus conferencias de presentación de la obra aquí comentada en Madrid y Barcelona, a quien agradezco el haberme facilitado este texto todavía inédito. 
sociedad civil partía de que los negocios entre particulares correspondían al ámbito exclusivo de la autonomía de la voluntad, bajo un régimen de consentimiento libre e informal.

Durante un dilatado período histórico los poderes públicos confiaron a la institución judicial la seguridad formal de los actos privados. Mientras que en el tejido social la función del cavere fue desarrollada por el notariado y el registro de la propiedad. La revolución burguesa contribuyó a potenciar estas instituciones por su inmediata relevancia para garantizar el derecho subjetivo de propiedad, clave de bóveda del orden jurídico liberal individualista. Pero la propia economía capitalista engendró un movimiento, el mercantilismo, que en su afán obsesivo por promover y agilizar las transacciones erosionó las garantías formales y, en primer término, las cautelares.

Entiende Mezquita del Cacho que las circunstancias aludidas no fueron ajenas a que en el Plan científico de división disciplinar del Derecho, elaborado en el pasado siglo, no se reservara espacio para el cavere. La evolución de la Teoría y la Metodología del Derecho quedó, de este modo, polarizada entre la elaboración conceptual y sistemática del Derecho propiciada por las derivaciones dogmáticas del respondere, y los desarrollos de las teorías argumentativas, neorretóricas, tópicas y revalorizadoras de la jurisprudencia que dimanan del postulare.

El cavere quedó, en gran medida, marginado de los cauces de elaboración científica y metodológica del Derecho. Sus funciones se consideraron meros remedios artesanales de técnica jurídica práctica, ligada a un ejercicio profesional privado, útiles para propiciar seguridad fáctica, pero carentes de entidad teórica y relegadas a una normación por vía reglamentaria. Se consagraba, así, un hiato entre la anatomía y la fisiología jurídicas, representadas por el Derecho material asumido «en estado de paz teórica», y la patología jurídica, metafóricamente cumplida por el Derecho procesal en cuanto marco de tratamiento de «la enfermedad del pleito». Y, por ende, resultaba postergada o infravalorada la inmunología e higiene jurídica, dirigida a «vacunar el cuerpo de las relaciones jurídicas privadas» para evitar conflictos, mediante procedimientos formales cautelares.

Una vez trazado, con ejemplar claridad, este sugerente mapa o itinerario de las tres grandes actividades en que se desglosa la experiencia jurídica, Mezquita del Cacho explicita su propósito; 
que no es otro que el de reivindicar las funciones de seguridad del cavere. El sistema cautelar viene considerado como un factor esencial para la garantía de los derechos e intereses legítimos, así como para evitar y ahorrar al Estado la contenciosidad excesiva y el consiguiente desastre institucional que produce. Por ello, reclama un espacio propio para el Sistema cautelar en la División disciplinar del Derecho. Tal espacio no debe quedar relegado al plano práctico, sino que debe implicar una renovada atención y elaboración científica de sus presupuestos y elementos estructurales. Se trata, en suma, de impulsar al respondere a que reflexione en profundidad sobre el cavere, para que se vea reducida la inflación perniciosa del postulare.

Al pasar revista a las causas que han podido incidir en esa marginación científica del sistema cautelar, Mezquita alude a la consabida tensión de la justicia respecto a la seguridad. La prioridad jurídica del valor de la justicia, así como la manipulación de la seguridad por los despotismos para legitimar sus legalidades represivas, han contribuido a fomentar la reticencia de quienes la afrontaban desde ópticas democráticas. Conviene, no obstante, advertir que los ataques más implacables contra la seguridad jurídica han procedido de los dos totalitarismos más caracterizados de nuestro siglo: el de signo nazi-fascista, que la ha impugnado por su significación conformista y antiheroica; y el comunista, que la reputa un producto de la ideología burguesa de orientación inequívocamente antiigualitaria.

Las reticencias, teóricas e ideológicas, frente a la seguridad jurídica se han ido desvaneciendo en la medida en que el constitucionalismo democrático ha situado en la cúspide de las funciones estatales la garantía de los derechos y libertades. Con ello, la seguridad jurídica no sólo se inmuniza frente al riesgo de su manipulación, sino que deviene un factor inevitable para el logro de los valores de la justicia y de la paz social. Desde ese enfoque debe contemplarse la rehabilitación de la seguridad jurídica operada por nuestra Constitución de 1978. Cuando se la invoca en el Preámbulo, en el art. 9.3 y en otros artículos del Título primero, se la contempla como un deber del Estado y se la funcionaliza para el logro de los derechos fundamentales. Así se evita el riesgo de su deformación o de su manipulación involutiva, y se la convierte en un valor y principio informador supernormativo de nuestro ordenamiento jurídico.

Desde esta perspectiva renovada la seguridad jurídica pierde su secular cariz antitético de la justicia, y se convierte en componente y promotora de justicia. La seguridad jurídica asume en nuestro ordenamiento el papel de valor y principio de triple proyección: 
inspiradora de las relaciones que en la esfera pública se dan entre el Estado y los ciudadanos; garante de la autonomía de la voluntad en las relaciones jurídico-privadas; e impulsora de la libertad civil en el terreno intermedio público/privado removiendo los obstáculos que desvirtúan la libertad entre desiguales, para lo que se requiere información plena y garantías cautelares frente a los riesgos de eventuales abusos. Uno de ellos, y no el menor, es el abuso procesal que se genera en los litigios entre partes de desigual capacidad de resistencia ante la demora y el riesgo de fallo injusto de las causas.

En el Estado social y democrático de Derecho el sistema cautelar, en cuanto uno de los principales cauces operativos de la seguridad jurídica, adquiere un importante cometido. Se trata de establecer unas garantías para equilibrar las situaciones fácticas de desigualdad que imponen los nuevos Leviatanes económicos (multinacionales, banca, macroempresas, lobbies de todo tipo...), a través de un sistema de protección preventista en contratos generales, de consumo de bienes y servicios básicos. El sistema cautelar puede así cumplir una primordial función de tutela de los consumidores, garantizando las formas frente a los grandes agentes económicos, que bajo el pretexto de la rapidez y eficacia, no dudan en avasallar cualquier interés social que se oponga a sus designios. Al cumplir esta función, el cavere no mengua la dinámica del tráfico, sino que la consolida por cifrarla en la confianza social. Suministra a los consumidores la dosis de respondere informativo y preventivo que precisan para hacer sus adquisiciones social y económicamente seguras. Y, por añadidura, contribuye a agilizar y descongestionar el postulare, casi siempre moratorio y dispendioso para los consumidores y usuarios.

En las cuestiones nodales y, por eso mismo, arduamente debatidas de la Filosofía y la Teoría del Derecho como lo es el concepto de seguridad jurídica, la exhaustividad resulta inalcanzable y las opciones de enfoque son ilimitadas. Por ello, el mérito y la utilidad de las investigaciones que afrontan esos conceptos y categorías se mide tanto por su capacidad para captar su persistente relevancia jurídica como para dar cuenta de los cambios de rumbo que periódicamente jalonan su andadura. La obra de Mezquita alcanza cumplidamente ambos cometidos. Supone un estudio monumental sobre el significado y alcance de la seguridad jurídica que, entre la infinitud de caminos para enjuiciarla, 
no deja de recorrer el suyo. A ello se orienta su empeño reconstructivo y, en cierto modo, rehabilitador de la función de la seguridad en la experiencia jurídica actual, realizado siguiendo el hilo conductor del sistema cautelar. Gracias a esa perspectiva se iluminan zonas tradicionalmente oscuras de la seguridad jurídica y las ya conocidas adquieren nueva evidencia.

Al propio tiempo, en este estudio se apuntan y compendian las principales transformaciones que hoy inciden en los vectores teóricos y prácticos de la seguridad jurídica. Desde premisas doctrinales distintas y sin alusión expresa a ellas, la investigación de Mezquita converge y se hace cargo de las principales inquietudes y proyecciones que hoy suscita la seguridad jurídica. Trataré de dar cuenta de alguna de ellas.

$$
* * *
$$

Con su habitual lucidez ha recordado Rudolf Wiethölter que el tránsito del Estado liberal al Estado social de Derecho supuso, entre otras cosas y no la de menor trascendencia, considerar al orden y la seguridad jurídica como el presupuesto, no la consecuencia de las libertades y derechos fundamentales. La seguridad deja así de concebirse como un mero apéndice resultante del ejercicio de las libertades individuales, para devenir condición necesaria de un sistema de derechos fundamentales plenamente garantizados. ${ }^{5}$

Esta llamada de atención ha potenciado una renovada óptica de encuadre de las relaciones entre seguridad y libertad. Ahora no tan sólo se ha superado la pretendida contradicción libertad/seguridad, al concebirse la seguridad como garante de la libertad. Se advierte en determinados empeños doctrinales del presente el propósito de rebasar la tradicional dimensión objetiva de la seguridad, en cuanto elemento informador del ordenamiento jurídico, para extenderla a la esfera subjetiva de los derechos fundamentales.

A ello, se orienta, por ejemplo, la reivindicación por parte de Josef Isensee de un derecho fundamental a la seguridad (Das Grundrecht auf Sicherheit). Este nuevo derecho fundamental no supondría sólo un límite a la actividad del Estado, una defensa frente a injerencias arbitrarias del poder público, fin prioritario atribuido por Wilhelm von Humboldt a la seguridad, sino que

${ }^{5}$ R. Wiethölter, Rechtswissenschaft, Helbing \& Lichtenhahn, Basel-Frankfurt, a.M., 2. ${ }^{\text {ed., }}$ 1986, págs. 165 y sigs. 
encauzaría la política estatal hacia la obtención de determinados bienes jurídicos directamente vinculados con la libertad y la igualdad de los ciudadanos. ${ }^{6}$

Nuestro sistema constitucional se hace eco de esa subjetivización de la seguridad jurídica. Por eso, tras consagrar su reconocimiento como valor (Preámbulo) y como principio informador del ordenamiento jurídico (art. 9.3), la consagra como un aspecto integrador, garantía de inmunidad, del derecho fundamental a la libertad personal (art. 17.1). Asimismo, nuestra Ley de leyes invoca expresamente a la seguridad como fin específico del compromiso de tutela de los consumidores y usuarios, a través de procedimientos eficaces, que incumbe a los poderes públicos (art. 51.1).

Estos nudos problemáticos de la seguridad hallan eco en la obra de Mezquita. Debe juzgarse del todo pertinente su propuesta hermenéutica de una lectura de la invocación a la seguridad jurídica contenida en el artículo 9.3 en clave del 9.2, propiciada por la identidad de la sedes materiae de positivación constitucional. Esta tesis vendría también avalada por una interpretación sistemática conectora del art. 9.3 con el artículo 1.1. En su virtud se entiende inherente al Estado social de Derecho «que la seguridad sirva a promover la igualdad real empezando por remover el obstáculo que deriva a este efecto del desequilibrio de poder en que en una sociedad neocapitalista se lleva a cabo la contratación sobre bienes de necesidad individual. La legislación de protección a usuarios y consumidores constituye muestra de dicho propósito...» (vol. I, pág. 246; vid. también, vol. II, págs. 197 y sigs.).

De forma análoga a la redimensión de las relaciones seguridad/libertad, se ha producido un replanteamiento de la tensión seguridad Justicia, tendente a superar los planteamientos basados en su pretendida antítesis irreductible. Entre estos últimos ha adquirido notoriedad, hasta el punto de constituir una cita clásica en la mayor parte de estudios sobre la seguridad, la expresión atribuida a Wolfgang Goethe de preferir sufrir la injusticia a soportar el desorden.

Esta tesis fue explicitada y ampliamente difundida por Gustava

\footnotetext{
${ }^{6}$ J. Isensee, Das Grundrecht auf Sicherheit, Walter de Gruyter, Berlin-New York, 1983, págs. 16 y sigs.
} 
Radbruch al situar la tensión justicia/seguridad como una de las antinomias fundamentales de la idea del Derecho. ${ }^{7}$ Luego fue formulada, con particular énfasis, por Francesco Carnelutti para quien el logro de la certeza entraña inevitablemente el sacrificio de la justicia, y la superación de la antítesis entre ambos valores sólo es posible al precio de admitir «l'infallibilità del legislatore». ${ }^{8}$

Frente a aquellos enfoques, Erhard Denninger ha sintetizado su diagnosis de la situación actual como un deslizamiento de la seguridad jurídica hacia la seguridad de los bienes jurídicos como justicia social: «Von der Rechtssicherheit zur Rechtsgütersicherheit als sozialer Gerechtigkeit». ${ }^{9}$

La aproximación entre seguridad y justicia se produce ahora a partir de una concreción de ambos valores. El primero deja de identificarse con la mera noción de legalidad o de positividad del Derecho, para conectarse inmediatamente con aquellos bienes jurídicos básicos cuyo «aseguramiento» se estima social y políticamente necesario. La justicia pierde su dimensión ideal y abstracta para incorporar las exigencias igualitarias y democratizadoras que informan su contenido en el Estado social de Derecho.

El principio de la protección de los bienes jurídicos (Rechtsgüterschutz) ha sido objeto de una amplia elaboración doctrinal por la Filosofía y la Teoría del Derecho (especialmente la penal) alemanas de nuestros días. En una extensa y documentada investigación debida a Knut Amelung se cifra en los ideales jurídicos de la Ilustración y, en especial en sus proyecciones en el pensamiento de Paul Johann Anselm Feuerbach de los inicios del siglo XIX, el planteamiento de esta tesis. En su formulación actual entraña una tendencia a funcionalizar los instrumentos de protección jurídica hacia el logro de bienes o valores jurídicos que se estiman imprescindibles para la convivencia social. ${ }^{10}$

${ }^{7}$ G. Radbruch, Filosofía del Derecho, trad. cast. de J. Medina Echevarría, Editorial Revista de Derecho Privado, Madrid, 1933, págs. 95 y sigs.

${ }^{8}$ F. Carnelutti, La certeza del diritto, incluido en el vol. de F. López de Oñate, ed. a cargo de G. Astuti, La certezza del diritto, Giuffrè, Milano, 1969, pág. 203. En este mismo trabajo alude expresamente a un «irriducibile contrasto tra la giustizia e la certezza del diritto», en pág. 199. Sobre el debate en relación con la certeza del Derecho suscitado en la doctrina jurídica italiana de la posguerra, cfr, mi libro, Iusnaturalismo y positivismo jurídico en la Italia moderna, Publicaciones del Real Colegio de España, Bolonia, 1970, págs. 132 y sigs.

${ }^{9}$ E. Denninger, «Der Präventions-Staat», en Kritische Justiz, 1988, núm. 1, pág. 1.

${ }^{10}$ K. Amelung, Rechtsgüterschutz und Schutz der Gesellschaft, Athenäum Vertag, Frankfurt a.M., 1972, págs. 4 y sigs., y 16 y sigs. 
$\mathrm{Su}$ relevancia positiva para una revisión de las funciones de la seguridad jurídica estriba en los siguientes aspectos:

$1 .^{\text {o }}$ Permite dotar de una base empírica a las garantías de seguridad, al vincularlas a la obtención de bienes jurídicos concretos (vida, libertad, salud, calidad de vida, seguridad en el tráfico...)

2. ${ }^{\circ}$ Contribuye a comprobar la eficacia del sistema de seguridad al ponerlo en relación con sus consecuencias en el plano de los bienes jurídicos a cuya tutela se dirige.

3. ${ }^{\circ}$ Legitima la función de la seguridad en el Estado social y democrático de Derecho como cauce operativo indispensable para la consecución de los grandes objetivos constitucionales. En particular, orienta la labor legislativa al establecimiento de técnicas de protección claras y justas de los bienes jurídicos.

Estos aspectos positivos no pueden velar algunos peligros que se denuncian como contrapunto de una concepción de la seguridad jurídica en cuanto seguridad de los bienes jurídicos. Entre los motivos críticos que se avanzan frente a esta tesis me parecen especialmente dignos de ser tomados en consideración los siguientes:

a) El carácter impreciso y equívoco de la propia noción de «bienes jurídicos». Con ella se corre el riesgo de caer en meras tautologías en las que se incurre cuando se define los bienes jurídicos como «bienes que incumben a todos», o «bienes que el Derecho debe proteger», etc. De otro lado, la propia amplitud de los objetos o bienes que hoy se juzgan merecedores de tutela jurídica que se extiende a temas tales como el medio ambiente, la protección de consumidores y usuarios, o la protección de los datos personales frente a abusos de la informática; así como la correlativa extensión de las situaciones o status subjetivos que se estiman dignos de protección y que tiende a incluir a cuantos poseen interés colectivos o difusos sobre esos bienes, amenaza con hacer «insegura» una seguridad basada en un concepto tan amplio y de perfiles tan difusos como el de bienes jurídicos.

b) Entre las imprecisiones que dimanan de la idea de los bienes jurídicos una de las más debatidas es la de si tales bienes se refieren a intereses individuales o sociales y colectivos. ${ }^{11}$ La identificación del bien jurídico con intereses puramente individuales

${ }^{11} \mathrm{Cfr}$. sobre la tensión entre concepciones individualistas y estatalistas, con especial referencia a los bienes jurídicos objeto de protección penal, cfr. W. Hassemer y F. Muñoz Conde, Introducción a la Criminología y al Derecho Penal, Tirant lo Blanch, Valencia, 1989, págs. 107 y sigs. 
parece difícilmente admisible en un Estado social y democrático de Derecho. No menos riesgo implica una versión transpersonalista o estatalista del bien jurídico que lo reduzca al interés de quienes detentan el poder político.

c) El peligro estatalista se ve potenciado por la conexión de la teoría del bien jurídico con la concepción preventiva del Estado. Así, para Erhard Denninger la conversión de la seguridad jurídica en seguridad de los bienes jurídicos representa la otra cara del proceso de conversión del Estado de Derecho en Estado de prevención (Preventions-Staat). En dicho orden político el Estado se legitima como garante de la justicia material mediante la producción de seguridad de unos bienes jurídicos, cuya definición el mismo se reserva. Así, so pretexto de la prevención de los bienes jurídicos, frente a hipotéticas o eventuales agresiones, recaba la adhesión incondicionada a sus medidas de control social restrictivas del ejercicio de la libertad. En la práctica política del Estado preventivo el ciudadano va dejando de ser paulatinamente sujeto de derechos fundamentales (Grundrechtssubjekt), para devenir mero destinatario de deberes fundamentales (Grundpflichtadressat). ${ }^{12}$

Para evitar los riesgos apuntados, entiendo que la seguridad de los bienes jurídicos debería responder a determinadas exigencias:

1. ${ }^{a}$ En primer término, la ambigüedad y equivocidad conceptual de esa categoría podría hallar un cauce de superación si se identifica con la noción, más precisa, de los valores constitucionalmente proclamados por los Estados de Derecho. A su vez, tales valores o bienes jurídicos hallarían así su concreción definitiva en el propio sistema de los derechos fundamentales. La actividad de las jurisdicciones constitucionales, en su labor hermenéutica y aplicadora de los derechos y libertades, constituye un elocuente y estimulante ejercicio continuado de delimitación práctica del contenido de los bienes jurídicos, así como de su compatibilización a través de la «ponderación de bienes» (Güterabwägung). ${ }^{13}$

2. ${ }^{\text {a }}$ La tensión entre la concepción individualista y transpersonalista de los bienes jurídicos halla precisamente su más acabado intento de solución en el Estado social de Derecho. En este tipo de organización política los derechos fundamentales, o sea,

\footnotetext{
${ }^{12}$ E. Denninger, op. cit., pág. 13.

${ }^{13}$ Vid. mi libro Derechos humanos, Estado de Derecho y Constitución, Tecnos, Madrid, 2. ${ }^{\mathrm{a}}$ ed., 1986, págs. 262 y sigs., y 286 y sigs.
} 
los bienes jurídicos tienden a posibilitar la integración plena y simultánea de las exigencias personales y sociales. Los derechos y libertades dejan de concebirse como un núcleo de garantías negativas dirigido a evitar las injerencias del poder público en la esfera privada, para devenir cauces positivos tendentes a remover los obstáculos de orden económico, social y cultural que impiden la plena realización de la libertad y la igualdad humanas. ${ }^{14}$

3. ${ }^{\mathrm{a}} \mathrm{La}$ función de seguridad de los bienes jurídicos, propia del Estado social de Derecho, puede eludir la involución autoritaria del Estado de prevención en la medida en que su estructura responda a los principios del pluralismo y la participación democrática. De este modo, será posible eludir el peligro de que el aseguramiento y la prevención de los bienes jurídicos actúe como un salvoconducto o una coartada legitimadora de cualquier tipo de política de seguridad del poder público. Se trata, en suma, de que en una sociedad democrática y pluralista los valores, bienes o derechos fundamentales no pueden ser el producto de la imposición arbitraria de un grupo ideológico, sino el resultado del consenso intersubjetivo edificado sobre supuestos procedimentalmente imparciales y a partir del sistema de necesidades radicales humanas. Se evita, de este modo, que la justicia social, que se identifica con la seguridad de los bienes jurídicos, corra el riesgo de transformarse en una forma de absolutismo ético-jurídico responsable de una tiranía de valores. ${ }^{15}$

La obra de Mezquita del Cacho no se adhiere, al menos de forma expresa, a las teorías germanas identificadoras de la seguridad con la seguridad de los bienes jurídicos. No obstante, es fácil advertir en sus páginas una reflexión que contiene notables puntos de coincidencia con las cuestiones que dimanan de esos planteamientos. Ese paralelismo de enfoque, desde culturas jurídicas distintas, revela la propia nodalidad problemática de cuanto hoy se debate al amparo de estos empeños reconstructivos y renovadores de la seguridad jurídica.

Un amplio apartado del tomo segundo (Capítulo 4, págs. 213-280) de la obra que suscita este comentario, se halla dedicado a exponer las virtualidades del sistema cautelar en el aseguramiento

${ }^{14}$ Ibíd., págs. 82 y sigs.; cfr. también mi volumen, Los derechos fundamentales, Tecnos, Madrid, 3. ${ }^{\mathrm{a}}$ ed., 1988, págs. 203 y sigs.

${ }^{15}$ Ibid., págs. 162 y sigs. 
de bienes jurídicos. Si bien, en el haber del planteamiento de Mezquita hay que anotar su precisa delimitación de la esfera orbital de incidencia de tales bienes, a través de una continuada referencia a los actos y estados jurídicos en los que se manifiesta en el plano de las relaciones privadas. Se evita, de este modo, que tal categoría se convierta en un «tipo ideal» impreciso y abstracto, bajo cuya rúbrica quepa todo.

La referencia constante a la dimensión preventiva del sistema cautelar, que constituye un evidente leitmotiv de la obra, tampoco implica la aceptación del modelo de Estado de prevención. La «dinámica preventista», reiteradamente invocada por Mezquita, no entraña una merma o infravaloración de las garantías formales del Estado de Derecho. Como expresamente puntualiza el Autor «la Forma sirve al fondo de las relaciones jurídicas, ya que constituye la ocasión de control de sus circunstancias y permite proyectar sobre ellas la luz de la libertad y de la equidad, alcanzándose seguridad sustancial a través de la certeza formal» (vol. 1, pág. 380).

La seguridad preventiva no se confunde, por tanto, con el valor de la justicia. Tiende hacia ella y favorece su consecución «pero no es en sí justicia, sino seguridad» (vol. 1, pág. 397). Por eso, el sistema de seguridad preventiva diseñado por Mezquita del Cacho nada tiene que ver con la hipóstasis de un valor absoluto y eterno legitimador de un Estado de prevención, peligro que acecha tras algunas formulaciones actuales de la tesis de la seguridad de los bienes jurídicos. La seguridad que dimana del sistema cautelar, que auspicia Mezquita, es siempre una garantía formal de carácter provisional y revisable. «Ningún sistema acumula tanta aptitud para conducir a la seguridad -en su opinión- como aquel que se construya desde la duda crítica (controles, calificaciones); aunque su status resultante siga siendo tan presuntivo y revisable (dudable, en suma); experiencia real que confirma la profunda verdad de aquel bello adagio de Samuel Beckett que proclama que entre todas las cosas seguras, ninguna es tan segura como la duda» (vol. I, pág. 398).

Los trabajos que abordan conceptos y categorías fundamentales de la Teoría del Derecho suelen responder a dos modelos diferenciados: el expositivo, que tiende a ofrecer la documentación básica del status quaestionis de la materia objeto de estudio, y puede también expresarse en forma de clasificaciones taxonómicas y tentativas metódicas de sistematización; y el creativo 
orientado hacia la propuesta de nuevas claves metódicas de afrontar los problemas, o a avanzar soluciones innovadoras. A un punto de encuentro entre ambos modelos pienso que responde la obra que suscita este comentario. En sus páginas se suceden y entremezclan, en proporción decisivamente correcta, la referencia amplia y rigurosa a la bibliografía básica de los distintos aspectos de la seguridad jurídica, con el avanzar de sus propias tesis y propuestas.

De primera impresión pudiera estimarse que la reiteración de excursos hacia temas colaterales, así como la profusión y amplitud de las notas a pie de página, ${ }^{16}$ diluyen el hilo discursivo de la obra. Pienso, por contra, que ese posible desbordamiento temático se ve compensado con usura por la riqueza de informaciones y entronques con asuntos concomitantes que, de continuo, se sugieren.

En cuestiones como la seguridad jurídica, cuyas implicaciones con otros institutos son ineliminables, la profundización vertical debe correr pareja con la horizontal. Se trata de evitar que el bosquejo de un contorno lineal y rectilíneo se logre a costa de eludir el dintorno; es decir, las fronteras de la realidad circundante que delimitan su sentido. De ahí, que el abundante aparato crítico-bibliográfico acoplado en esas citas no sea una mera digresión teórica, sino la referencia obligada para seguir el itinerario intelectual del Autor. Es más, gracias a ese generoso esfuerzo por hacer transparentes los soportes de su investigación un amplio catálogo de materiales bibliográficos, relacionados mediata o inmediatamente con la seguridad jurídica, han pasado a engrosar el acervo de fuentes y pistas doctrinales puestas al servicio de cuantos en el seno de nuestra cultura jurídica deseen prolongar el estudio de esa noción insoslayable de la Teoría del Derecho.

Admira el esfuerzo intelectual que estos dos amplios tomos sobre la Seguridad jurídica y el sistema cautelar están denunciando. La riqueza de documentación, la penetración crítica y, suum cuique, su cuidada presentación. Todo ello honra tanto a su

${ }^{16}$ A la relevancia de las notas bibliográficas para la investigación científica del Derecho se han referido P. Häberle y A. Blankenagel, «Fussnoten als Instument der Rechts-Wissenschaft», en Rechtstheorie, 1988, núm. 19, págs. 116 y sigs.

\section{DOXA 7 (1990)}


Autor como a cuantos han hecho posible su publicación. 